\title{
Synergistic Use of Satellite Laser Altimetry and Shuttle Radar Topography Mission DEM for Estimating Ground Elevation Over Mountainous Vegetated Areas
}

\author{
Wen Liu and Qi Chen
}

\begin{abstract}
Topography is fundamental to numerous environmental studies for understanding Earth surface processes. With a near global coverage, satellite lidar Geoscience Laser Altimeter System (GLAS) onboard Ice, Cloud, and land Elevation Satellite (ICESat) provides valuable terrain elevation information via Gaussian decomposition of its waveforms. It is commonly assumed that the lowest decomposed Gaussian peak corresponds to terrain surface. Although this assumption is valid over flat areas with sparse canopy, it might be problematic over sloped areas with surface objects. This letter proposes a new algorithm to estimate ground elevation over mountainous vegetated areas using GLAS data in conjunction with Shuttle Radar Topographic Mission Digital Elevation Models (SRTM DEMs). It was found that incorporating SRTM DEM can reduce the bias of the mean ground elevation estimates by up to 1-3 m. This letter also suggests that more research is needed for improving ground elevation estimates over mountainous areas with relatively open canopy.
\end{abstract}

Index Terms-Airborne lidar, Gaussian decomposition, ground elevation, Ice, Cloud, and land Elevation Satellite (ICESat)/Geoscience Laser Altimeter System (GLAS), Shuttle Radar Topographic Mission C-band Digital Elevation Model (SRTM DEM).

\section{INTRODUCTION}

$\mathbf{H}$ IGH-QUALITY topographic data are crucial for understanding various land surface processes [1]-[3]. The Geoscience Laser Altimeter System (GLAS) onboard the Ice, Cloud, and land Elevation Satellite (ICESat) is a powerful sensor capable of providing ground elevation information with high precision and nearly global coverage [4], [5]. The ground elevation retrieved from the GLAS offers critical information not only on bare Earth [6] but also on canopy vertical structure [7]-[9]. For example, the GLAS has been used to derive canopy height [10]-[13], which can be calculated as the difference between canopy top and ground elevation [7], [14], [15].

Gaussian decomposition is a common technique to calculate ground elevation from the GLAS. This method decomposes GLAS waveforms into several individual Gaussian distributions so that terrain and surface object elevation information within

Manuscript received December 23, 2011; revised June 2, 2012 and July 17, 2012; accepted July 20, 2012. Date of publication August 28, 2012; date of current version November 24, 2012.

The authors are with the Department of Geography, University of Hawaii at Manoa, Honolulu, HI 96822 USA (e-mail: wenl@hawaii.edu; qichen@ hawaii.edu).

Color versions of one or more of the figures in this paper are available online at http://ieeexplore.ieee.org.

Digital Object Identifier 10.1109/LGRS.2012.2210536 the GLAS shot can be reconstructed [16]. It is usually assumed that one of the Gaussian peaks, particularly the lowest Gaussian peak, represents the ground information [6], [7], [14], [17], [18]. In an area where it is flat with relatively open canopy, the lowest Gaussian peak can match well with the ground [14]. However, over sloped variable terrains, there might be multiple Gaussian peaks associated with reflections from the ground surface. Some studies [6], [7] found that, over mountainous areas, the lowest Gaussian peak is generally lower than the actual mean ground elevation, but the second or third to the lowest peak could be closer to the ground [6]. These studies indicated that the correspondence between the Gaussian peak and the ground is strongly dependent on the terrain and vegetation conditions; the information from GLAS Gaussian decomposition alone is insufficient to determine which Gaussian peaks represent signals from the ground. To address this problem, it is pressing to determine how Gaussian peaks correspond to the ground and to develop a method to use these peaks to estimate the ground elevation.

In this letter, we introduce a novel algorithm for retrieving mean ground elevation $z_{m}$ through a synergist use of GLAS data and Shuttle Radar Topographic Mission C-band Digital Elevation Models (SRTM DEMs). Airborne-lidar-derived ground elevations $z_{v}$ are used to evaluate the estimated $z_{m}$ value. Both 30- and 90-m SRTM DEMs are tested to investigate the effects of DEM spatial resolutions on estimating $z_{m}$.

\section{Study AREA And DATA}

\section{A. Study Sites}

This study is conducted at five individual vegetated mountainous sites located in the Northwest United States. The first site, located in Mendocino, CA, is covered by coniferous forests consisting of upland redwood, pygmy cypress dwarf, northern mixed chaparral, and bishop pine forests. The second site, located in Lewis, WA, is covered by coniferous forests in early to late successional stages. The third site, located in Santa Clara, $\mathrm{CA}$, is primarily dominated by oak woodland and California annual grassland. The fourth site is located in Iredell, NC, with major land covers of pasture, deciduous forests, and shrubs. The fifth site, located in the Appalachians range (traversing the counties of Watauga, Avery, Burke, and McDowell), NC, is dominated by deciduous forests with a minority of evergreen 


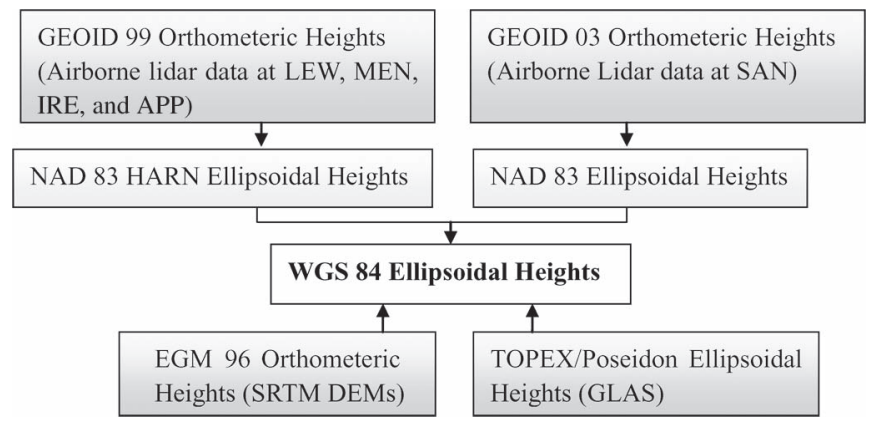

Fig. 1. Flowchart of vertical datum conversions.

trees, pasture, and deciduous trees [6], [7]. These five sites are denoted as LEW, MEN, SAN, IRE, and APP sites hereinafter. Among the first three, the LEW and MEN sites are dominated by conifers that share similar mean tree height $(\sim 35 \mathrm{~m})$ and canopy cover $(\sim 0.9)$, while the SAN site has much shorter vegetation $(\sim 10.7 \mathrm{~m}$ on average) and lower canopy cover $(\sim 0.27)[7]$.

\section{B. Data}

Three data sets are applied in our analyses, i.e., the GLAS, the SRTM DEM, and the airborne-lidar data. The GLAS data were obtained from the National Snow and Ice Data Center (http://nsidc.org/data/icesat/), which archives and distributes 15 products of level-1 (L1) and level-2 (L2) laser altimetry and atmospheric GLAS data. In this letter, we used GLA 01 (L1A Altimetry Data) and GLA 14 (L2 Land Surface Altimetry) from the release 28 [7].

The SRTM C-band DEMs of 1 and 3 arc-second (approximately 30 and $90 \mathrm{~m}$ ) were downloaded from the Earth Resources Observation and Science Center, U.S. Geological Survey. All SRTM data are stored in $1^{\circ}$ tiles in raster format.

The airborne-lidar data of the LEW and MEN sites were acquired by the Airborne Lidar Terrain Mapping (ALTM) System in February 2003, while the data of the SAN site were obtained by the Optech ALTM 3100 lidar system during flights in April-May 2006 [7]. The airborne-lidar data of the IRE and APP sites were collected in early 2003 by EarthData International using the Azimuth Aeroscan system [6].

\section{Data Preprocessing}

The three data sets were originally in different horizontal and vertical datum. The airborne-lidar data and the SRTM were in orthometic height (referring to geoid surface), while the GLAS was in ellipsoid height (referring to an ellipsoid). To be consistent, both the GLAS and the airborne-lidar data are converted into the same horizontal datum as that of the SRTM data in the World Geodetic System 1984 (WGS 84). Elevations of all data were converted to the WGS 84 ellipsoidal height (see Fig. 1).

The ground elevation validations $z_{v}$ from airborne-lidar data were processed by the Toolbox for Lidar Data Filtering and Forest Studies (TIFFS) software package via automatically identifying terrain returns and interpolating them into a $1-\mathrm{m}$

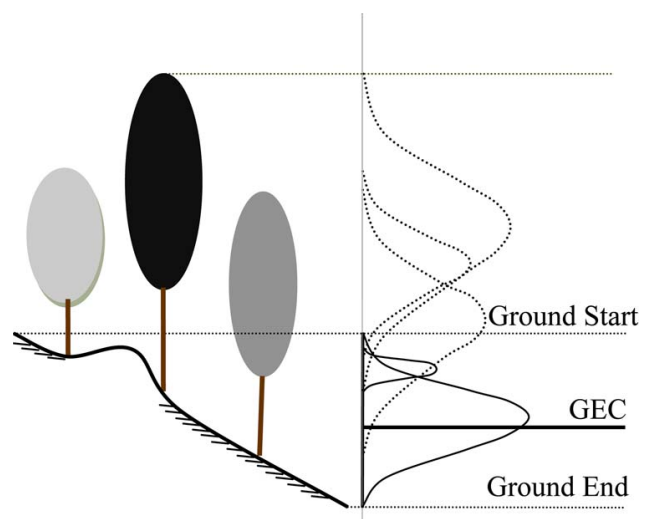

Fig. 2. Finding of the ground vertical extent and ground Gaussians. The vertical range between the ground start and the ground end is the ground vertical extent. The GEC is the referencing point to locate ground vertical extent. Within the ground vertical extent, (black line) the two decomposed Gaussian components are identified as ground Gaussians. Gaussians in gray dot line are above-ground Gaussians.

raster DEM [6], [7], [19]. The derived $z_{v}$ value was quantitatively assessed by a manually edited National Aeronautics and Space Administration DEM with the statistical difference of $0.01 \pm 0.73 \mathrm{~m}$ (mean \pm standard derivation) [7]. Furthermore, slopes at GLAS shots were extracted from the fused SRTM DEM tiles by the surface analyst tools in ArcGIS.

Extra steps were taken to remove GLAS shots affected by bad weather or saturation [7]. As a result, a total of 66, 200, 134, 692, and 611 good GLAS shots remain at the LEW, MEN, SAN, IRE, and APP sites, respectively.

\section{METHODS}

\section{A. Gaussian Decomposition of the GLAS Waveform}

The shape of the transmitted laser pulse of the GLAS shows a near-Gaussian distribution [5]. A received waveform is the result of the convolution of the transmit pulse and the differential cross sections, which provides the theoretic basis of the Gaussian decomposition of the GLAS waveform [16]. To minimize the influences of the high frequency noise, the received waveforms were first smoothed by the transmit pulse before the initial determination of waveform parameters. The smoothed waveform can be modeled as the sum of Gaussian components [20], as shown in the following functions:

$$
\begin{aligned}
W(t) & =\sum_{m=1}^{N} W_{m}(t) \\
W_{m}(t) & =A_{m} e^{\frac{-\left(t-\mu_{m}\right)^{2}}{2 \sigma_{m}^{2}}}
\end{aligned}
$$

where $W(t)$ is the intensity of the received smoothed waveform at time $t, W_{m}(t)$ is the intensity contribution of the $m$ th Gaussian, $N(N<=6)$ is the number of Gaussian components, $A_{m}$ is the amplitude of the $m$ th Gaussian, $\mu_{m}$ is the centroid of the $m$ th Gaussian, and $\sigma_{m}$ is the standard deviation (1/e halfwidth) of the $m$ th Gaussian. $A_{m}, \mu_{m}$, and $\sigma_{m}$ are recorded in the product GLA 14. An illustration of Gaussian composition is shown in Fig. 2. 
TABLE I

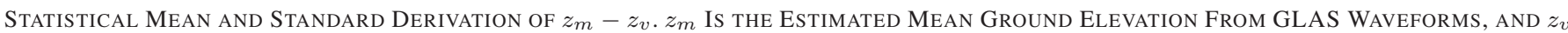
Is the Ground Elevation Derived From Airborne Lidar. Method 1 IndiCATEs Results When Ground Elevation CEnters ARE Calculated Based on (7) (9). Method 2 Indicates the Results When (10) Is Used to Detect the Ground Elevation Center and (2-5pks) Refers to the Number of Gaussian Peaks Included in the Method. Method 3 Indicates the Results When $z_{m}$ Is Calculated as the Elevation of the Gaussian Peak of Higher Amplitude Among the Lowest Two. The Best Results of EACH Site Are SET In Bolded And Underlined

\begin{tabular}{|c|c|c|c|c|c|c|c|c|c|c|c|}
\hline \multirow{4}{*}{$\begin{array}{c}\text { Study sites } \\
\text { (shots) }\end{array}$} & \multicolumn{11}{|c|}{$z_{m}-z_{v}$ (METER) } \\
\hline & \multicolumn{5}{|c|}{ 30-m SRTM DEM } & \multicolumn{5}{|c|}{ 90-m SRTM DEM } & \multirow{3}{*}{$\begin{array}{l}\text { No DEM } \\
\text { Method3 }\end{array}$} \\
\hline & \multirow{2}{*}{ Method1 } & \multicolumn{4}{|c|}{ Method2 } & \multirow{2}{*}{ Method1 } & \multicolumn{4}{|c|}{ Method2 } & \\
\hline & & $2 p k s$ & 3pks & 4pks & $5 p k s$ & & $2 p k s$ & 3pks & 4pks & $5 p k s$ & \\
\hline LEW (66) & $-0.6 \pm 8.4$ & $6.7 \pm 8.2$ & $9.7 \pm 8.3$ & $11.6 \pm 8.7$ & $12.1 \pm 8.6$ & $\underline{-0.6 \pm 8.2}$ & $7.0 \pm 8.3$ & $9.8 \pm 8.2$ & $11.9 \pm 8.4$ & $12.6 \pm 8.4$ & $3.4 \pm 8.1$ \\
\hline MEN (200) & $-1.1 \pm 7.0$ & $6.0 \pm 8.1$ & $8.4 \pm 8.0$ & $9.7 \pm 8.1$ & $10.7 \pm 8.3$ & $-1.6 \pm 7.2$ & $6.0 \pm 8.3$ & $8.3 \pm 8.0$ & $9.7 \pm 8.1$ & $10.7 \pm 8.3$ & $3.2 \pm 7.7$ \\
\hline SAN (134) & $-3.6 \pm 6.0$ & $-1.7 \pm 4.3$ & $-1.0 \pm 3.1$ & $-0.8 \pm 3.0$ & $0.7 \pm 3.1$ & $-3.6 \pm 6.0$ & $-2.0 \pm 4.6$ & $-1.0 \pm 3.0$ & $-0.7 \pm 3.0$ & $\underline{-0.6 \pm 3.0}$ & $-4.4 \pm 5.5$ \\
\hline IRE (692) & $-1.5 \pm 8.2$ & $0.3 \pm 7.7$ & $2.7 \pm 7.5$ & $4.4 \pm 7.8$ & $5.4 \pm 8.2$ & $-2.2 \pm 8.1$ & $\underline{-0.1 \pm 7.7}$ & $2.5 \pm 7.4$ & 4. $3 \pm 7.8$ & $5.5 \pm 8.1$ & $-1.0 \pm 7.5$ \\
\hline APP (611) & $\underline{-0.2 \pm 3.3}$ & $1.0 \pm 3.9$ & $2.6 \pm 5.2$ & $3.6 \pm 6.1$ & $4.4 \pm 7.0$ & $-0.4 \pm 3.2$ & $1.0 \pm 3.8$ & $2.7 \pm 5.0$ & $3.7 \pm 5.9$ & $4.4 \pm 6.7$ & $-0.4 \pm 3.2$ \\
\hline
\end{tabular}

\section{B. Algorithm of Calculating $z_{m}$}

The principle of our algorithm of calculating the mean ground elevation $z_{m}$ is to first detect the Gaussian peaks corresponding to the terrain ground, which are called ground Gaussians (e.g., the black Gaussians in Fig. 2), and to then average elevations of all ground Gaussians, i.e.,

$$
\begin{aligned}
z_{m} & =\frac{\sum_{i=1}^{N} \text { flag }_{i} \times E_{i}}{\sum_{i=1}^{N} \text { flag }_{i}} \\
\text { flag }_{i} & =0, \text { nonground Gaussian } \\
\text { flag }_{i} & =1, \text { ground Gaussian }
\end{aligned}
$$

where $N(1<=N<=6)$ is the number of Gaussians, flag $_{i}$ is the flag of the $i$ th to the lowest Gaussian indicating whether it is a ground Gaussian, and $E_{i}$ is the elevation of the $i$ th to the lowest Gaussian peak. Ground Gaussians are those within ground vertical extent, a vertical range defining the starting and the ending of ground signals. The size of the ground vertical extent, denoted as GES, can be calculated as follows [21]:

$$
\operatorname{GES}=(m)=\tan (\text { slope }) * \operatorname{GLASFootPrintSize}(m) .
$$

Along with the size, a referencing point (e.g., its center, starting point, or ending point) is needed to determine the exact location of the ground vertical extent. We choose the center as its referencing point and call it ground extent center (GEC). In this letter, we propose two different methods (denoted as methods 1 and 2) to automatically identify GECs.

In method 1, GECs are selected between the lowest two Gaussian peaks using

$$
\begin{aligned}
\mathrm{GEC} & =E_{1}, \quad \text { when } A_{1}>A_{2} \text { OR } \Delta \mathrm{VE}_{1} \leq \Delta \mathrm{VE}_{2} \\
\mathrm{GEC} & =E_{2}, \quad \text { when } A_{1}<A_{2} \text { AND } \Delta \mathrm{VE}_{1}>\Delta \mathrm{VE}_{2} \\
\Delta \mathrm{VE}_{i} & =\left|\mathrm{GW}_{i}-\mathrm{GES}\right|
\end{aligned}
$$

where $A_{i}$ and $\mathrm{GW}_{i}$ are the amplitude and the width of the $i$ th to the lowest Gaussian, and $\Delta \mathrm{VE}_{i}$ is the size difference between the $i$ th Gaussian width and the ground vertical extent.

In method 2, GECs are chosen among the lowest $N$ peaks by

GEC $=E_{i} ;$ where $S_{i}$ is the maximum

among the lowest $N$ Gaussians

where $S_{i}$ is the area of the $i$ th to the lowest Gaussian. $N$ ranges from 2 to 5 , and $2 \leq i \leq N$. Corresponding to the number of lowest $N$ Gaussian peaks included in method 2, the specific algorithms are denoted as $2 \mathrm{pks}, 3 \mathrm{pks}, 4 \mathrm{pks}$, and $5 \mathrm{pks}$, respectively (see Table I).

To compare with methods from previous studies, another set of $z_{m}$ values are calculated as the elevations of the Gaussian peak with higher amplitude between the lowest two Gaussians [6]. This is denoted as method 3.

\section{RESULTS}

The $z_{m}$ values estimated by various algorithms were compared against $z_{v}$ with the statistics of their differences $\left(z_{m}-z_{v}\right.$, or bias of $z_{m}$ ) listed in Table I, where the algorithm with the smallest bias at each study site is set in bold and underlined. The LEW, MEN, and APP sites had the $z_{m}$ estimate of the smallest bias from method 1 , while the best results of $z_{m}$ were from method 2 of $5 \mathrm{pks}$ at the SAN site and from method 2 of 2 pks at the IRE site.

Method 1 generally performed well at all study sites. Compared with method 3 , method 1 was capable of reducing the bias of $z_{m}$ by about 1-3 $\mathrm{m}$ at the LEW, MEN, and SAN sites; at the IRE and APP sites, the biases of $z_{m}$ from method 1 were comparable with those from method 3 with their values less than $1.5 \mathrm{~m}$ (see Table I).

Method 2 outperformed method 1 at the woodland (SAN) and IRE sites (see Table I) but failed to give acceptable estimates of $z_{m}$ at two coniferous sites and at the APP site. By using method 2, the $z_{m}$ bias tended to decrease with the 
increasing number of Gaussians at the SAN site, while the bias was minimized method 2 of 2 pks and then increased when more numbers of Gaussians were included at the APP site. The smallest biases were $-0.6 \mathrm{~m}$ at the SAN site and $-0.1 \mathrm{~m}$ at the APP site.

\section{Discussions}

Our results indicated that method 1 generally improves estimates of $z_{m}$ compared with those from method 3. This is mainly attributed to the two improvements in our algorithm, i.e., using the SRTM DEM to aid the identification of ground peaks and averaging all identified ground Gaussian peaks instead of using one single selected Gaussian peak for calculating mean ground elevation.

We also found that the spatial resolutions ( 30 versus $90 \mathrm{~m}$ ) of SRTM DEMs had limited impacts on estimates of $z_{m}$. The bias of $z_{m}$ differs less than $0.2 \mathrm{~m}$ between the two SRTM DEMs for all algorithms and sites, except when method 1 is used at the LEW site. We found that this is very likely related to the small differences in ground vertical extents derived from 30- and 90-m SRTM DEMs. Mean slope differences are $2.4^{\circ}$ at the IRE site, $1.5^{\circ}$ at the APP site, and less than $0.3^{\circ}$ at the LEW, MEN, and SAN sites, respectively. Note that the 30-m SRTM DEM is unavailable at the global scale while the 90-m SRTM DEM is. This has important implications to potentially expand our algorithm to other places using the 90-m SRTM DEM without sacrificing much accuracy compared with the application of the 30-m SRTM DEM.

Individual GLAS shots were analyzed to better understand biases in our $z_{m}$ estimates. When method 1 was used, the biases of $z_{m}$ could be as large as $-20-23$ and $-22-31 \mathrm{~m}$ at the LEW and MEN sites, respectively, while biases of $z_{m}$ by method 2 of 5 pks ranged from -12.8 to $13.96 \mathrm{~m}$ at the SAN site. After visually exploring the features of the vegetation distributions and the topographical variations from Google Earth at these specific shots, we found that the overestimation of $z_{m}$ usually happens when the canopy is dense so that the laser energy hardly penetrates into lower elevation, which makes the detection of ground Gaussian peaks difficult. Fig. 3(a) shows such an example shot where the estimated ground vertical extent (thus $z_{m}$ ) is much higher than $z_{v}$. The underestimation of $z_{m}$ frequently happens over locations with steep topography. We originally suspected that this is caused by the coarse spatial resolution DEM, given that the 30- or 90-m SRTM will flatten valleys and peaks in topography, leading to underestimated slopes and thus underestimated ground vertical extent according to (6). However, no obvious improvements were found after we used the slope derived from airborne-lidar data to derive the ground elevation extent size (GES). Therefore, the large bias of $z_{m}$ [see Fig. 3(b) for an example] is mainly caused by the imprecise estimation of the ground vertical extent center (GEC), which is more difficult to be determined over steep slopes.

Since the accuracy of $z_{m}$ estimates is largely determined by algorithms of detecting GECs, we further compared the elevations of individual Gaussian peaks to $z_{v}$ and identified the Gaussian peak closest to $z_{v}$ for each shot. We found that, at the LEW, MEN, and APP sites, 90\% of the closest Gaussians are from the lowest two peaks (see Table II), which ensures
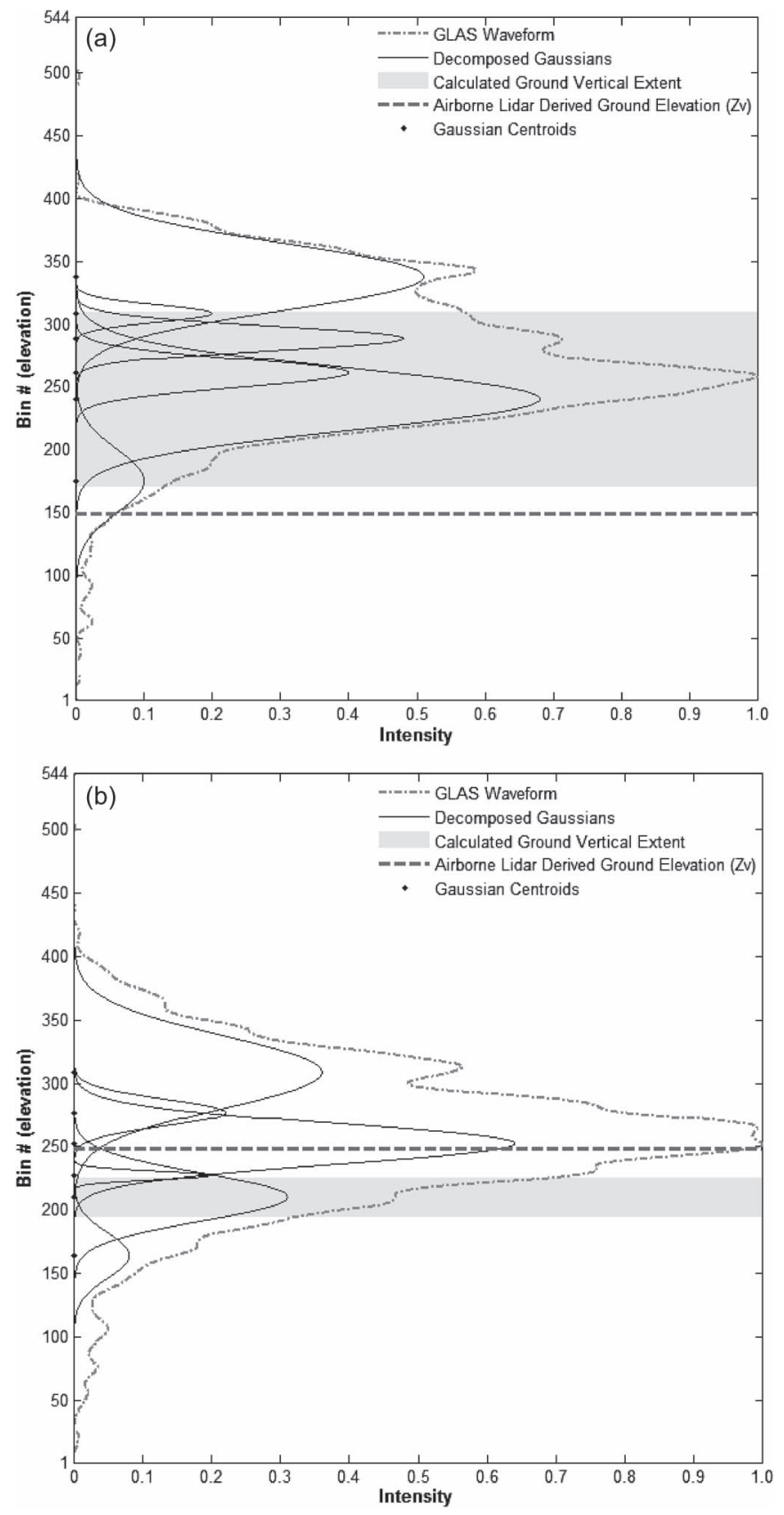

Fig. 3. Biased $z_{m}$ estimates. (a) Shot where the calculated $z_{m}$ value (within the ground vertical extent, i.e., the shaded area) is much higher than $z_{v}$. (b) Shot where the calculated $z_{m}$ value is much lower than $z_{v}$.

TABLE II

Percentage of the $n$ Th Gaussian Peaks That Are the Closest One Among Six Decomposed Gaussians to Ground ELEVATION VALIDATIONS $z_{v}$

\begin{tabular}{lllllll}
\hline & $1^{\text {st }} \mathrm{PK}$ & $2^{\text {nd }} \mathrm{PK}$ & $3^{\text {rd }} \mathrm{PK}$ & $4^{\text {th }} \mathrm{PK}$ & $5^{\text {th }} \mathrm{PK}$ & $6^{\text {th }} \mathrm{PK}$ \\
\hline LEW & $47.0 \%$ & $42.4 \%$ & $9.1 \%$ & $1.5 \%$ & $0 \%$ & $0 \%$ \\
MEN & $42.0 \%$ & $47.5 \%$ & $8.5 \%$ & $1.5 \%$ & $0 \%$ & $0.5 \%$ \\
SAN & $10.45 \%$ & $27.6 \%$ & $32.8 \%$ & $19.4 \%$ & $9.0 \%$ & $0.75 \%$ \\
IRE & $21.24 \%$ & $38.73 \%$ & $26.01 \%$ & $11.71 \%$ & $2.02 \%$ & $0.29 \%$ \\
APP & $59.4 \%$ & $34.53 \%$ & $5.24 \%$ & $0.65 \%$ & $0.16 \%$ & $0 \%$ \\
\hline
\end{tabular}

method 1 would be optimal to describe GECs. At the two remaining sites (particularly SAN), a relatively high percentage of the closest Gaussians were from the third or fourth to the 
lowest peaks (see Table II). This explains the large biases when method 1 was used to estimate $z_{m}$ at these two sites. In general, we found that method 1 performed the best at sites of relatively dense canopy (LEW, MEN, and APP), while method 2 performed better at sites of sparse and open canopy (SAN and IRE). Mountainous areas with open canopy pose the most significant challenge because it is unclear how many of Gaussian peaks should be included in method 2 to achieve the best results, which calls for more future research to improve ground elevation estimation over these areas.

\section{REFERENCES}

[1] S. Clarke and K. Burnett, "Comparison of digital elevation models for aquatic data development," Photogramm. Eng. Remote Sens., vol. 69, no. 12, pp. 1367-1375, Dec. 2003.

[2] T. M. Neeson, A. M. Gorman, P. J. Whiting, and J. F. Koonce, "Factors affecting accuracy of stream channel slope estimates derived from geographical information systems," North Amer. J. Fisheries Manage., vol. 28, no. 3, pp. 722-732, Jun. 2008.

[3] P. Tarolli, J. R. Arrowsmith, and E. R. Vivoni, "Understanding earth surface processes from remotely sensed digital terrain models," Geomorphology, vol. 113, no. 1/2, pp. 1-3, Dec. 2009.

[4] H. J. Zwally, B. Schutz, W. Abdalati, J. Abshire, C. Bentley, A. Brenner, J. Bufton, J. Dezio, D. Hancock, D. Harding, T. Herring, B. Minster, K. Quinn, S. Palm, J. Spinhirne, and R. Thomas, "ICESat's laser measurements of polar ice, atmosphere, ocean, and land," J. Geodyn., vol. 34, no. 3/4, pp. 405-445, Oct./Nov. 2002.

[5] D. J. Harding and C. C. Carabajal, "ICESat waveform measurements of within-footprint topographic relief and vegetation vertical structure," Geophys. Res. Lett., vol. 32, pp. L21S10-1-L21S10-4, Oct. 2005.

[6] Q. Chen, "Assessment of terrain elevation derived from satellite laser altimetry over mountainous forest areas using airborne lidar data," ISPRS J. Photogramm. Remote Sens., vol. 65, no. 1, pp. 111-122, Jan. 2010.

[7] Q. Chen, "Retrieving vegetation height of forests and woodlands over mountainous areas in the Pacific Coast region using satellite laser altimetry," Remote Sens. Environ., vol. 114, no. 7, pp. 1610-1627, Jul. 2010.

[8] M. A. Lefsky, "A global forest canopy height map from the moderate resolution imaging spectroradiometer and the geoscience laser altimeter system," Geophys. Res. Lett., vol. 37, pp. L15401-1-L15401-5, Aug. 2010.

[9] M. Simard, N. Pinto, J. B. Fisher, and A. Baccini, "Mapping forest canopy height globally with spaceborne lidar," J. Geophys. Res., vol. 116, pp. G04021-1-G04021-12, Nov. 2011.
[10] M. A. Lefsky, D. J. Harding, M. Keller, W. B. Cohen, C. C. Carabajal, F. D. Espirito-Santo, M. O. Hunter, and R. de Oliveira, "Estimates of forest canopy height and aboveground biomass using ICESat," Geophys. Res. Lett., vol. 32, no. 22, pp. L22S02-1-L22S02-4, Nov. 2005.

[11] M. A. Lefsky, M. Keller, Y. Pang, P. B. de Camargo, and M. O. Hunter, "Revised method for forest canopy height estimation from geoscience laser altimeter system waveforms," J. Appl. Remote Sens., vol. 1, p. 013537 , Sep. 2007.

[12] J. A. B. Rosette, P. R. J. North, and J. C. Suarez, "Vegetation height estimates for a mixed temperate forest using satellite laser altimetry," Int. J. Remote Sens., vol. 29, no. 5, pp. 1475-1493, Mar. 2008.

[13] M. Simard, V. H. Rivera-Monroy, J. E. Mancera-Pineda, E. Castaneda-Moya, and R. R. Twilley, "A systematic method for 3D mapping of mangrove forests based on shutter radar topography mission elevation data, ICESat/GAS waveforms and field data: Application to Cienaga Grande de Santa Marta, Colombia," Remote Sens. Environ., vol. 112, no. 5, pp. 2131-2144, May 2008.

[14] A. L. Neuenschwander, T. J. Urban, R. Gutierrez, and B. E. Schutz, "Characterization of ICESat/GLAS waveforms over terrestrial ecosystems: Implications for vegetation mapping," J. Geophys. Res., vol. 113 no. G2, pp. G02S03-1-G02S03-18, Apr. 2008.

[15] G. Sun, K. J. Ranson, D. S. Kimes, J. B. Blair, and K. Kovacs, "Forest vertical structure from GLAS: An evaluation using LVIS and SRTM data," Remote Sens. Environ., vol. 112, no. 1, pp. 107-117, Jan. 2008.

[16] W. Wagner, A. Ullrich, V. Ducic, T. Melzer, and N. Studnicka, "Gaussian decomposition and calibration of a novel small-footprint full-waveform digitizing airborne laser scanner," ISPRS J. Photogramm. Remote Sens., vol. 60 , no. 2, pp. 100-112, Apr. 2006.

[17] M. A. Hofton, J. B. Minster, and J. B. Blair, "Decomposition of laser altimeter waveforms," IEEE Trans. Geosci. Remote Sens., vol. 38, no. 4 , pp. 1989-1996, Jul. 2000.

[18] H. Duong, R. Lindenbergh, N. Pfeifer, and G. Vosselman, "ICESat fullwaveform altimetry compared to Airborne laser scanning altimetry over the Netherlands," IEEE Trans. Geosci. Remote Sens., vol. 47, no. 10, pp. 3365-3378, Oct. 2009

[19] Q. Chen, "Airborne lidar data processing and information extraction," Photogramm. Eng. Remote Sens., vol. 73, no. 2, pp. 109-112, Feb. 2007.

[20] A. C. Brenner, H. J. Zwally, C. R. Bentley, B. M. Csatho, D. J. Harding, M. A. Hofton, J. B. Minster, L. A. Roberts, J. L. Saba, R. H. Thomas, and $\mathrm{D}$. Yi, "Derivation of range and range distributions from laser pulse waveform analysis for surface elevations, roughness, slope, and vegetation heights," in Geoscience Laser Altimeter System (GLAS)—Algorithm Theoretical Basis Document. Greenbelt, MD: GSFC, 2003.

[21] S. Lee, W. Ni-Meister, W. Yang, and Q. Chen, "Physically based vertical vegetation structure retrieval from ICESat data: Validation using LVIS in White Mountain National Forest, New Hampshire, USA," Remote Sens. Environ., vol. 115, no. 11, pp. 2776-2785, Nov. 2011. 